\title{
The Gaia-ESO Survey: Inhibited extra mixing in two giants of the open cluster Trumpler 20?^
}

R. Smiljanic ${ }^{1}$, E. Franciosini ${ }^{2}$, S. Randich ${ }^{2}$, L. Magrini ${ }^{2}$, A. Bragaglia ${ }^{3}$, L. Pasquini $^{4}$, A. Vallenari ${ }^{5}$, G. Tautvaišiené ${ }^{6}$, K. Biazzo $^{7}$, A. Frasca $^{7}$, P. Donati ${ }^{3,8}$, E. Delgado Mena ${ }^{9}$, A. R. Casey ${ }^{10}$, D. Geisler ${ }^{11}$, S. Villanova ${ }^{11}$, B. Tang ${ }^{11}$, S. G. Sousa ${ }^{9}$, G. Gilmore ${ }^{10}$, T. Bensby ${ }^{12}$, P. François ${ }^{13,14}$, S. E. Koposov ${ }^{10}$, A. C. Lanzafame ${ }^{15}$, E. Pancino ${ }^{2,16}$,

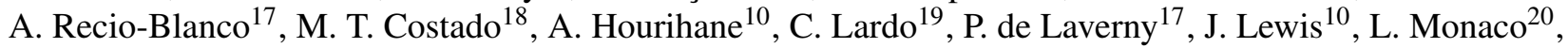
L. Morbidelli ${ }^{2}$, G. G. Sacco ${ }^{2}$, C. C. Worley ${ }^{10}$, S. Zaggia ${ }^{5}$, and S. Martell ${ }^{21}$

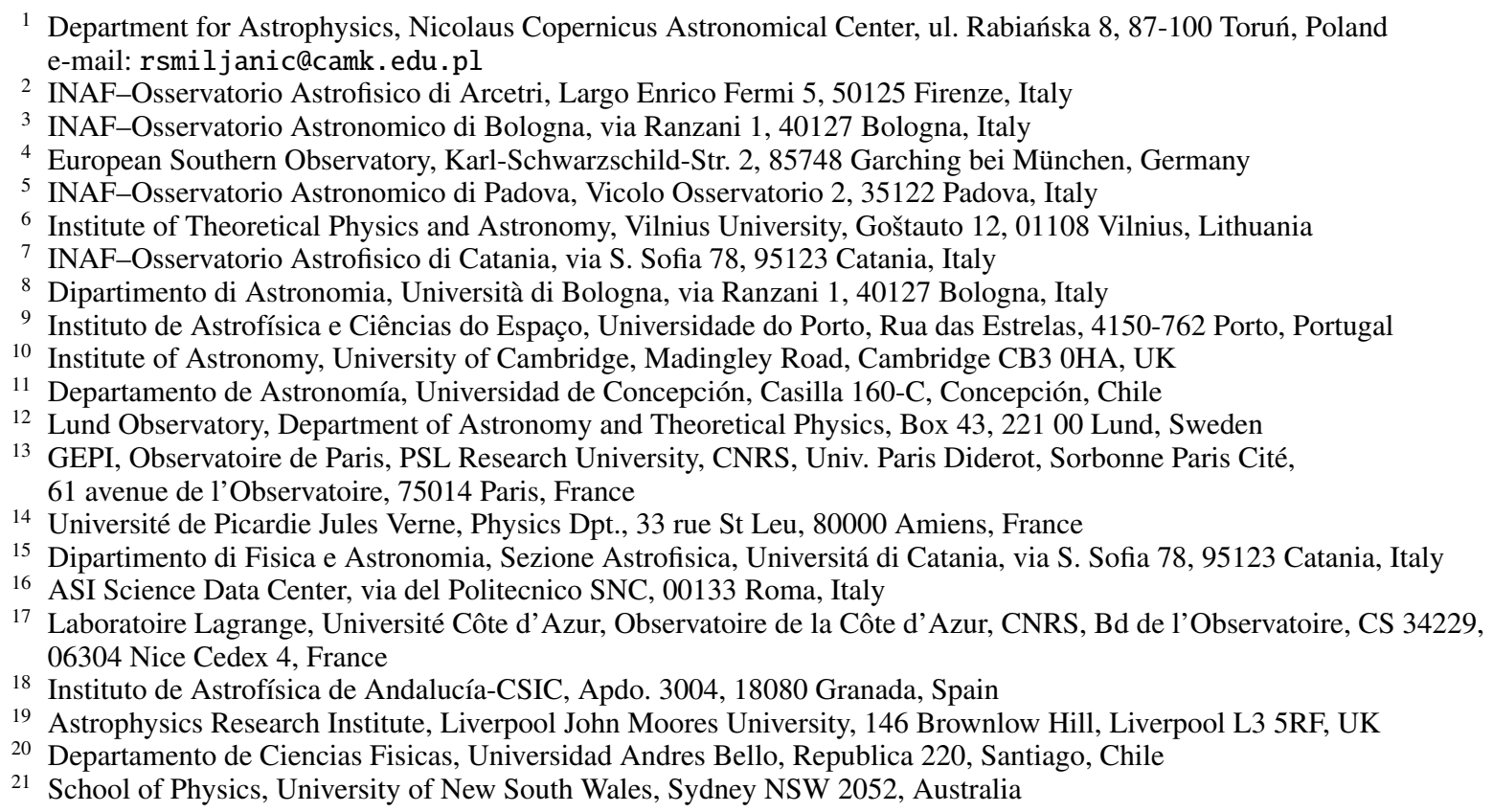

Received 16 March 2016 / Accepted 29 April 2016

\section{ABSTRACT}

\begin{abstract}
Aims. We report the discovery of two Li-rich giants, with $A(\mathrm{Li}) \sim 1.50$, in an analysis of a sample of 40 giants of the open cluster Trumpler 20 (with turnoff mass $\sim 1.8 M_{\odot}$ ). The cluster was observed in the context of the Gaia-ESO Survey.

Methods. The atmospheric parameters and Li abundances were derived using high-resolution UVES spectra. The Li abundances were corrected for nonlocal thermodynamical equilibrium (non-LTE) effects.

Results. Only upper limits of the $\mathrm{Li}$ abundance could be determined for the majority of the sample. Two giants with detected $\mathrm{Li}$ turned out to be Li rich: star MG 340 has $A(\mathrm{Li})_{\text {non-LTE }}=1.54 \pm 0.21$ dex and star MG 591 has $A(\mathrm{Li})_{\text {non-LTE }}=1.60 \pm 0.21$ dex. Star MG 340 is on average $~ 0.30$ dex more rich in Li than stars of similar temperature, while for star MG 591 this difference is on average $\sim 0.80$ dex. Carbon and nitrogen abundances indicate that all stars in the sample have completed the first dredge-up.

Conclusions. The $\mathrm{Li}$ abundances in this unique sample of 40 giants in one open cluster clearly show that extra mixing is the norm in this mass range. Giants with $\mathrm{Li}$ abundances in agreement with the predictions of standard models are the exception. To explain the two Li-rich giants, we suggest that all events of extra mixing have been inhibited. This includes rotation-induced mixing during the main sequence and the extra mixing at the red giant branch luminosity bump. Such inhibition has been suggested in the literature to occur because of fossil magnetic fields in red giants that are descendants of main-sequence Ap-type stars.
\end{abstract}

Key words. stars: abundances - stars: evolution - stars: late-type - open clusters and associations: individual: Trumpler 20

\section{Introduction}

\footnotetext{
^ Based on observations collected at the European Organisation for Astronomical Research in the Southern Hemisphere under ESO programme 188.B-3002 (The Gaia-ESO Public Spectroscopic Survey).
}

Although not well understood, the phenomenon of Li-rich giants seems to be ubiquitous as they have been observed in different environments: open clusters, globular clusters, metal-rich 
and metal-poor field stars, the Galactic bulge, and also in dwarf galaxies (see, e.g., Hill \& Pasquini 1999; Gonzalez et al. 2009; Kumar et al. 2011; Ruchti et al. 2011; Kirby et al. 2012, 2016; D'Orazi et al. 2015, and references therein).

Lithium-rich giants are usually defined as those that, after the first dredge-up, have $A(\mathrm{Li}) \geq 1.50 \mathrm{dex}$. This limit is the postdredge-up Li abundance of a low-mass star according to standard evolutionary models, i.e., those models that include only convection as a mixing mechanism. The first Li-rich giant was a fortuitous discovery by Wallerstein \& Sneden (1982). Subsequent searches have shown that these stars comprise about $1-2 \%$ of red giants (Brown et al. 1989; Pilachowski et al. 2000). Charbonnel \& Balachandran (2000) suggested that these objects appear at the luminosity bump of the red giant branch (RGB) or at the early-asymptotic giant branch (AGB) for low- and intermediatemass stars, respectively. Other recent results preferably classify these objects as core helium-burning giants (Kumar et al. 2011; Monaco et al. 2014; Silva Aguirre et al. 2014). Nevertheless, some Li-rich giants have been found throughout the RGB (see, e.g., Alcalá et al. 2011; Monaco et al. 2011; Martell \& Shetrone 2013).

Lithium-rich giants have other noteworthy characteristics that add complexity to the puzzle. Some present a far-infrared excess, suggesting a connection with enhanced mass loss (de la Reza et al. 1996). This mass-loss event can also explain the observation of complex organic and inorganic compounds detected in the infrared spectra of some Li-rich giants (de la Reza et al. 2015). In a few cases, the presence of circumstellar material has been confirmed by polarimetry (Pereyra et al. 2006). Nevertheless, not all Li-rich giants have an infrared excess (see, e.g., Jasniewicz et al. 1999; Bharat Kumar et al. 2015; Rebull et al. 2015).

Fekel \& Balachandran (1993) proposed a connection between Li enrichment, fast rotation, and chromospheric activity. Lithium-rich giants seem to be more common among fast rotating stars ( $50 \%$; see, e.g., Drake et al. 2002). A strong magnetic field was detected in one Li-rich giant by Lèbre et al. (2009). Further examples of Li-rich, fast-rotating, active giants exist (e.g., Reddy et al. 2002; Kriskovics et al. 2014; Strassmeier et al. 2015).

In addition, a few Li-rich giants hosting planets have been found (e.g., Adamów et al. 2012, 2014). As proposed by Siess \& Livio (1999), surface Li enrichment could be caused by planet engulfment, which also causes spin-up, magnetic field generation, and shell ejection. However, planet accretion would create a ${ }^{9} \mathrm{Be}$ enhancement that has never been detected in Li-enriched objects (de Medeiros et al. 1997; Castilho et al. 1999; Melo et al. 2005; Pasquini et al. 2014) with the exception of one Ftype dwarf in the open cluster NGC 6633 (Ashwell et al. 2005). Alternatively, planet engulfment could activate internal Li production and induce its mixing to the surface (Denissenkov \& Weiss 2000).

Indeed, the properties of many Li-rich giants discovered within the Gaia-ESO Survey (Gilmore et al. 2012; Randich \& Gilmore 2013) seem to be consistent with those of giants that engulfed close-in giant planets before evolving up the RGB (Casey et al. 2016). However, a small fraction of cases still require alternative explanations. Here, we report the discovery of two Li-rich giants that could be examples of such an alternative formation channel in the open cluster Trumpler 20, which is a system of $\sim 1.66 \mathrm{Gyr}$ in age and $[\mathrm{Fe} / \mathrm{H}]=+0.17$ (Donati et al. 2014).

This paper is organized as follows. In Sect. 2 we briefly describe the data used here, the analysis, and the properties of our sample. In Sect. 3 we discuss how extra mixing is needed to
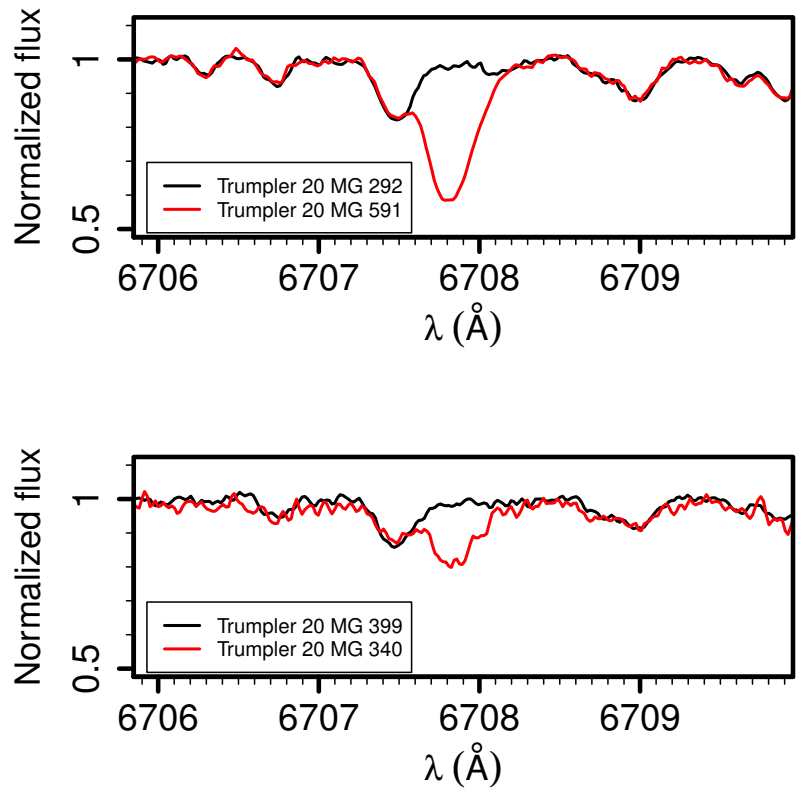

Fig. 1. Comparison of the spectra around the Li $6708 \AA$ line between the Li-rich giants and giants with similar atmospheric parameters.

explain the surface Li abundances of the majority of the sample. In Sect. 4 we discuss the two Li-rich giants and the possibility that they have avoided extra-mixing mechanisms. Finally, Sect. 5 summarizes our findings and suggests new observations that could support our interpretation of the Li enrichment in these two giants.

\section{Data, analysis, and sample properties}

The high-resolution $(R \sim 47000)$ UVES (Ultraviolet and Visual Echelle Spectrograph, Dekker et al. 2000) spectra of 42 targets in Trumpler 20 were obtained in the context of the Gaia-ESO Survey. Data reduction is described in Sacco et al. (2014). Basic information on the observed giants is available in Table A.1.

The atmospheric parameters and abundances (see Table A.2) are part of the fourth Gaia-ESO internal data release (iDR4). The spectra were analyzed using the Gaia-ESO multiple pipelines strategy (Smiljanic et al. 2014) with an updated methodology (Casey et al., in prep.).

Membership was assigned using the radial velocities (RVs) as in Donati et al. (2014). Likely members (40 giants in total) are those with RV within three standard deviations of the cluster average $\left(\overline{\mathrm{RV}} \pm \sigma=-40.2 \pm 1.3 \mathrm{~km} \mathrm{~s}^{-1}\right)$. One star is a subgiant ${ }^{1}$ and one a probable nonmember (or binary) with deviant $\mathrm{RV}^{2}$.

The Li abundances were determined from the $6708 \AA$ line. In Fig. 1, we compare the Li $6708 \AA$ lines of the two Li-rich giants to those of stars with similar atmospheric parameters to illustrate the $\mathrm{Li}$ enhancement. Corrections for nonlocal thermodynamical equilibrium (non-LTE) effects were applied using the grid of Lind et al. (2009). For the giants, the corrections range from 0.14 dex to 0.32 dex, depending on the atmospheric parameters.

The color magnitude diagram (CMD) of Trumpler 20 is shown in Fig. 2. The photometry is originally from Carraro et al. (2010) corrected for differential reddening by Donati et al. (2014). The uncertainties in the magnitudes are

\footnotetext{
1 Trumpler 20 MG 430. The numbering system we adopt is that defined in McSwain \& Gies (2005).

2 Trumpler $20 \mathrm{MG} 894$ with $\mathrm{RV}=-35.3 \mathrm{~km} \mathrm{~s}^{-1}$.
} 


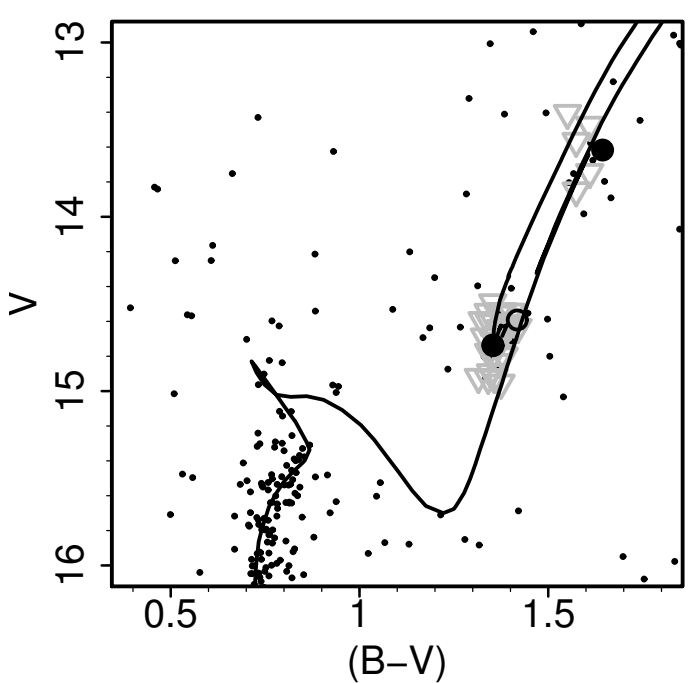

Fig. 2. Color magnitude diagram of Trumpler 20. Only stars within 3 of the cluster center are shown. The two Li-rich giants are shown as filled circles, other giants with Li detections as open circles, giants with Li upper limits as gray triangles, and the remaining stars in the field as dots. The solid line is an isochrone from Bressan et al. (2012) with age = $1.66 \mathrm{Gyr}$ and $[\mathrm{Fe} / \mathrm{H}]=+0.17$, which is the best fit to the photometric data as determined by Donati et al. (2014).

$\sim 0.02-0.05$ mag. A noticeable feature in this CMD is the extended clump region of the cluster. Trumpler 20 is well known for its peculiar extended clump region (see Carraro et al. 2010; Platais et al. 2012; Donati et al. 2014). This feature is probably caused by the presence of two distinct clumps; the fainter clump comprises stars massive enough to start core He-burning in nondegenerate conditions and the brighter clump comprises stars with slightly lower mass that have been through the Hecore flash (see, e.g., Girardi 1999; Girardi et al. 2000).

Figure 3 shows the sample in the $T_{\text {eff }}-\log g$ plane. The group of giants with lower $\log g$ are either at the luminosity bump of the RGB or at the early-AGB, as both stages are within the error bars of the parameters in Fig. 3 and are hard to separate in the CMD of Fig. 2. We can be more confident about the evolutionary state of the group of giants with higher $\log g$ because of their chemical abundances.

The evolutionary stage of the stars is an important source of information to interpret their $\mathrm{Li}$ abundances, as a high $\mathrm{Li}$ abundance could just be indicating that the giant is actually at the bottom of the RGB (e.g., Böcek Topcu et al. 2015). Nevertheless, the $\mathrm{C}$ and $\mathrm{N}$ abundances of the giants demonstrate that they have all completed the standard first dredge-up. As shown in Fig. 4, according to the models of Lagarde et al. (2012), giants of 1.5 and $2.0 M_{\odot}$ after the dredge-up have $C / N \sim 1$, as do all the giants in our sample. Giants at the bottom of the RGB with $T_{\text {eff }} \sim 5000 \mathrm{~K}$ would be in the stage before the end of the first dredge-up and thus would instead have $C / N>3$. We can thus safely conclude that i) all the giants with $T_{\text {eff }} \sim 5000 \mathrm{~K}$ are in the red clump and not on the RGB and ii) that all the brightest and coolest giants have completed the Li dilution expected during the first dredge-up. The $\mathrm{C}$ and $\mathrm{N}$ abundances of the Trumpler 20 giants were discussed in Tautvaišienè et al. (2015).

\section{Extra mixing in the majority of the giants}

Lithium abundances have been extensively used as a tracer of mixing processes, as $\mathrm{Li}$ is rapidly destroyed in $(p, \alpha)$ reactions at

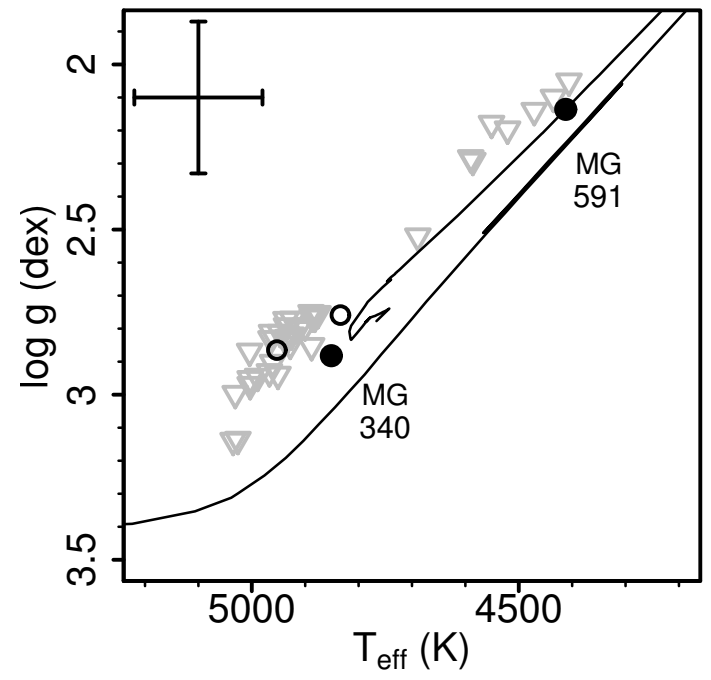

Fig. 3. Trumpler 20 giants in the $T_{\text {eff }}-\log g$ plane. The two Li-rich giants are shown as filled circles, other giants with Li detections as open circles, and giants with $\mathrm{Li}$ upper limits as gray triangles. The solid line is an isochrone from Bressan et al. (2012) of $1.66 \mathrm{Gyr}$ in age and $[\mathrm{Fe} / \mathrm{H}]=$ +0.17 , which is the best fit to the photometric data (by Donati et al. 2014). A typical error bar $\left( \pm 120 \mathrm{~K}\right.$ and $\pm 0.23 \mathrm{dex}$ for $T_{\text {eff }}$ and $\log g$, respectively) is shown in the top left.

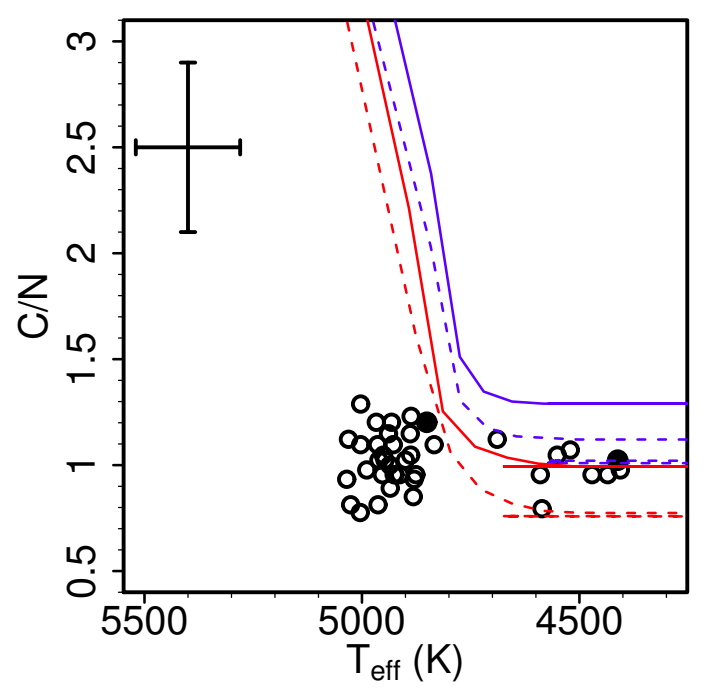

Fig. 4. $\mathrm{C} / \mathrm{N}$ ratio as a function of $T_{\text {eff }}$. Solid lines are the predictions of standard models and dashed lines of models with rotation-induced mixing and thermohaline mixing (Lagarde et al. 2012). Lines in blue and red are for solar metallicity stars of $1.5 M_{\odot}$ and $2.0 M_{\odot}$, respectively. The two Li-rich giants are shown as full circles.

temperatures above $2.5 \times 10^{6} \mathrm{~K}$ (Greenstein \& Richardson 1951). Thus, Li only survives in the outermost layers of a star. As stars evolve to the RGB, the convective envelope deepens and $\mathrm{Li}$ from the surface is diluted.

Figure 5 shows the Li abundances as a function of the effective temperatures $\left(T_{\text {eff }}\right)$, for all cluster members, in comparison with the models of Lagarde et al. (2012). The bulk of the stars fall in between the standard and extra-mixing models (solid and dashed lines, respectively). However, care is needed in interpreting the plot because of the evolutionary state of the stars.

The solid and dashed lines in Fig. 5 are predictions for first ascent RGB stars and not for clump giants. As we showed above, our giants with $T_{\text {eff }} \sim 5000 \mathrm{~K}$ are clump giants and not first ascent RGB stars. The observations should not be compared to 


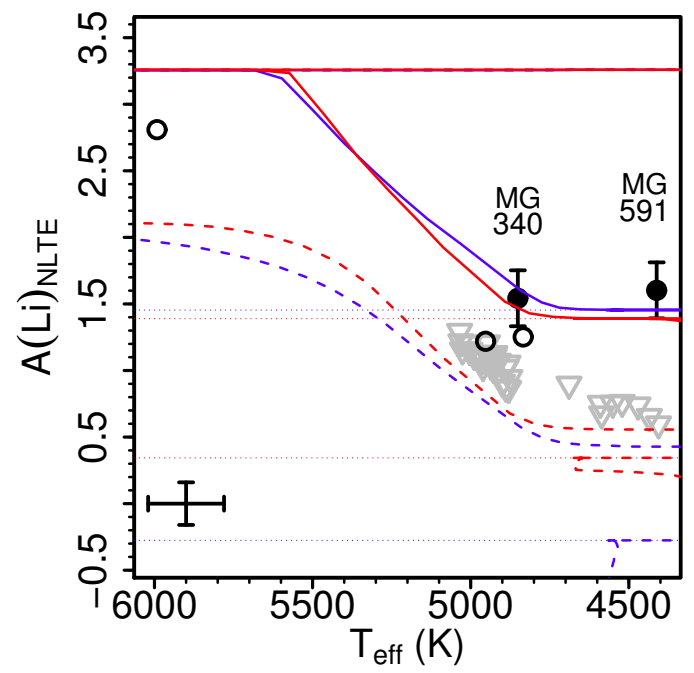

Fig. 5. Lithium abundance as a function of $T_{\text {eff }}$. The two Li-rich giants are shown as filled circles, other giants with Li detections as open circles, and giants with Li upper limits as gray triangles. Solid lines are the predictions of standard models and dashed lines of models with rotation-induced mixing and thermohaline mixing (Lagarde et al. 2012). Lines in blue and red are for solar metallicity stars of $1.5 M_{\odot}$ and $2.0 M_{\odot}$, respectively. The dotted lines are included as an eye guide to the $\mathrm{Li}$ abundance level of clump giants in the models (but we note here that the clump phase does not span the $T_{\text {eff }}$ range of the dotted lines in the plot).

this region of the models, but rather to the $A(\mathrm{Li})$ level of core-He burning giants (the dotted lines). The clump giants have Li upper limits on average of about 0.3 dex below the two top dotted lines (standard models). For lower temperatures, the second group of stars has also Li upper limits well below the prediction of the standard models.

The clear exceptions to that are the two Li-rich giants, Trumpler 20 MG 340 and 591. This agrees with the findings of Brown et al. (1989) that giants with $A(\mathrm{Li}) \sim 1.50$ in agreement with standard models are a minority. Here, we are able to confirm this result in a large sample of giants of the same age, same initial chemical composition, and very similar masses. The enhanced Li depletion/dilution seen in the majority of the giants of Trumpler 20 is well documented in the literature (e.g., Lèbre et al. 1999; Pasquini et al. 2001, 2004), although the mechanism behind this extra mixing is still under debate.

We note another possible outlier, star MG 505, with $A(\mathrm{Li})_{\text {non-LTE }}=1.25 \pm 0.21$. However, because within the errors its abundance agrees with the highest upper limit at its temperature, we do not consider it among those that agree with the standard models.

\section{Inhibited extra mixing in two giants}

Star MG 340 has $A(\mathrm{Li})_{\text {non-LTE }}=1.54 \pm 0.21$ and $T_{\text {eff }}=4851 \mathrm{~K}$, while in eight other stars with $T_{\text {eff }}=4850 \pm 50 \mathrm{~K}$ there is one detection at $A(\mathrm{Li})_{\text {non-LTE }}=1.25$ and seven upper limits below $A(\mathrm{Li})_{\text {non-LTE }} \sim 1.05$. Star MG 591 has $A(\mathrm{Li})_{\text {non-LTE }}=1.60 \pm 0.21$ and $T_{\text {eff }}=4412 \mathrm{~K}$, while seven other stars with $T_{\text {eff }}<4600 \mathrm{~K}$ have upper limits below $A(\mathrm{Li})_{\text {non-LTE }} \sim 0.76$.

These two Li-rich giants do not show any additional chemical peculiarity when compared to the other cluster giants. Both stars seem to be single, but we do not have multiple epoch spectra to exclude long period companions. All sample giants seem to be slow rotators: $v \sin i<4.0 \mathrm{~km} \mathrm{~s}^{-1}$. Therefore, the
$\mathrm{Li}$ enhancement is probably not connected to rotation in the sense seen by Lèbre et al. (2006) and Carlberg et al. (2012). These authors found that in a given sample of giants, those with higher $\mathrm{Li}$ abundance tend to be those giants that are rotating faster, however, our giants might seem to be slow rotators because of an unfavorable line of sight. Slow rotation also argues against, but does not fully exclude, planet accretion with transfer of angular momentum as the source of the Li enhancement (see also Carlberg et al. 2016; Delgado Mena et al. 2016). Thus, external pollution as advocated by Casey et al. (2016) to explain other Li-rich giants discovered within the Gaia-ESO Survey seems unlikely in our case.

Internal $\mathrm{Li}$ production was also suggested to explain Lirich giants. Fresh $\mathrm{Li}$ production might occur in the stellar interior through the ${ }^{7} \mathrm{Be}$ mechanism (Cameron \& Fowler 1971). However, it is still unknown which transport mechanism would bring ${ }^{7} \mathrm{Be}$, which decays to ${ }^{7} \mathrm{Li}$, to the surface (Sackmann \& Boothroyd 1999; Palacios et al. 2001). Charbonnel \& Balachandran (2000) argued that Li-rich giants were preferentially found at the bump and the early-AGB, and connected the $\mathrm{Li}$ enrichment with an extra-mixing process that activates at these evolutionary stages. For low-mass stars at the bump, the extra mixing would be connected to the H-burning shell that is moving outward and removes the molecular weight discontinuity left by the receding convective layer. In intermediate-mass stars, the extra mixing would take place at the early-AGB when the convective envelope deepens again.

The two Li-rich clump giants found by Silva Aguirre et al. (2014), with $A(\mathrm{Li})=2.71$, and Monaco et al. (2014), with $A(\mathrm{Li})=3.75$, showed that the above scenario is at least incomplete. For clump giants, the Li enrichment could be connected to the He flash, following an episode of $\mathrm{H}$ injection in deeper high temperature regions (Mocák et al. 2011).

Because of the large surface convective layers of giants, the observed Li surface enrichment is likely to be short lived. A Lirich low-mass giant that appears at the bump should not remain $\mathrm{Li}$ rich during its evolution to the clump. More likely, the two Li-rich giants discovered here have been freshly created during or close to their current evolutionary stages. Thus, to explain our Li-rich giants with internal $\mathrm{Li}$ production would require a combination of the two distinct scenarios above. One Li-rich giant would be created by mixing induced by the He flash, the other would be created by extra mixing at the bump. It also seems an odd coincidence that we would happen to observe both Li-rich giants at the moment in which their Li abundances are very close to the value expected by standard models.

While internal Li production could indeed be required to explain abundances above the meteoritic value $(A(\mathrm{Li})>3.0)$, this is perhaps not necessary to explain stars MG 340 and 591 . We instead suggest that their higher $\mathrm{Li}$ abundance is the result of the inhibition of extra-mixing processes. Without extra mixing, their surface $\mathrm{Li}$ abundance is at the level predicted by standard models. In addition, this single scenario would be able to explain both giants regardless of their different evolutionary states.

If this suggestion is correct, two instances of extra mixing must have been affected. The first is the extra Li dilution beyond the predicted first dredge-up dilution, which is observed as the star leaves the main sequence toward the RGB. Observationally, it is well known that an extra mixing causes the Li dilution to start earlier than predicted by standard models (e.g., Lèbre et al. 1999; Pasquini et al. 2001, 2004). This is because rotationinduced mixing creates a Li-free region inside real stars that is larger than predicted by these models (Palacios et al. 2003). In the two Li-rich giants, rotation-induced mixing must have been 
weak and the Li dilution proceeded as expected by standard models.

The second extra-mixing event to be avoided is the event taking place at the luminosity bump of the RGB (see Lagarde et al. 2012, and references therein). The luminosity bump (see, e.g., Christensen-Dalsgaard 2015) happens at the RGB of low-mass stars when the hydrogen-burning shell reaches the composition discontinuity left behind by the first dredge-up. The current best candidate for the mechanism behind this extra mixing seems to be thermohaline mixing (Charbonnel \& Zahn 2007b), although there are discussions about the physical properties and efficiency of this mechanism (e.g., Maeder et al. 2013; Garaud \& Brummell 2015; Lattanzio et al. 2015, and references therein).

Star MG 340 in the extended clump of the cluster is likely to be a low-mass star. As discussed by Girardi (1999), in such an extended clump, the less massive stars are actually the brighter stars. Both the CMD in Fig. 2 and the $T_{\text {eff }}-\log g$ diagram in Fig. 3 seem to indicate that MG 340 belongs to the group of brighter giants. Thus, in our scenario, for it to keep an unaltered $\mathrm{Li}$ surface abundance, thermohaline mixing must have been inhibited. On the other hand, star MG 591 is either on the bump or on the early-AGB. If on the early-AGB, then it is an intermediate-mass star that does not go through both the He-flash and the bump phase. However, it would still need to inhibit thermohaline mixing at the early-AGB (see Lagarde et al. 2012, and references therein). If on the bump, it either avoided thermohaline mixing or did not activate it yet. All other giants with similar $T_{\text {eff }}$ and $\log g$ have lower Li abundances. This could indicate that star MG 591 is also after the moment where thermohaline mixing becomes efficient. As pointed out by the referee, however, at this phase stars ascend and reascend the RGB, crossing the same $T_{\text {eff }}$ and $\log g$ region three times. Therefore, it is plausible that at least one star among the group at the bump has not yet activated thermohaline mixing. If this is the case, star MG 591 would not be an Li-rich giant, but a normal giant in a stage before extra mixing was activated.

Extra-mixing inhibition is not a new idea. Based on carbon isotopic ratios, ${ }^{12} \mathrm{C} /{ }^{13} \mathrm{C}$, Charbonnel \& Do Nascimento (1998) estimated that about $4 \%$ of low-mass giants do not experience extra mixing on the RGB. Charbonnel \& Zahn (2007a) suggested that extra mixing is avoided by giants that are descendant from Ap-type main-sequence stars. In these stars, fossil magnetic fields would be able to inhibit thermohaline mixing. Modern estimates of the percentage of Ap stars with respect to nonmagnetic A-type stars are between 1.7-3.5\% (North 1993; Power et al. 2007). This is consistent with finding one or two stars in our sample of 40 giants of Trumpler 20 (a fraction of 2.5 or $5 \%$ ). In addition, the stellar mass of our giants is within the mass range of Ap stars ( $\sim 1.5$ to $3.6 M_{\odot}$, Power et al. 2007).

\section{Summary}

In this work, we presented the discovery of two Li-rich giants in the open cluster Trumpler 20. These two stars were identified in an analysis of a sample of 40 giants for which high-resolution spectra were obtained with UVES in the context of the GaiaESO public spectroscopic survey. This provides a unique large sample of giants that have the same age, same initial chemical composition, and very similar masses. The Li abundances in this sample clearly demonstrate that extra mixing is the norm in stars in this mass range. Giants with $\mathrm{Li}$ abundances in agreement with the predictions of standard models are the exception.

To explain the two Li-rich giants, we suggest that all instances of extra-mixing processes have been inhibited. Because of that, the surface Li abundance in these two stars remained at the level predicted by standard stellar evolution models, i.e., $A(\mathrm{Li}) \sim 1.50$. We argue that the fraction of Li-rich giants found in our sample is consistent with these giants being evolved counterparts of magnetic Ap-type dwarfs. In this case, the extramixing processes could have been inhibited by the action of magnetic fields, as suggested by Charbonnel \& Zahn (2007a).

Other explanations seem less likely, although they cannot be fully excluded. Extra Li from the accretion of external material should be accompanied by accretion of angular momentum, but there is no evidence of fast rotation in the two giants. Because the two Li-rich giants have different evolutionary stages, internal $\mathrm{Li}$ production would require two different mechanisms to bring the fresh Li to the surface. The extra-mixing inhibition hypothesis would instead be able to explain both giants at the same time.

Additional observations could help in providing extra support to our suggested scenario, or they could help to disprove it. First, if no extra mixing took place, the carbon isotopic ratio should be close to the prediction of standard models, ${ }^{12} \mathrm{C} /{ }^{13} \mathrm{C} \sim$ 30. We could not determine the carbon isotopic ratio because the region around $8000 \AA$, containing the $\mathrm{CN}$ bands preferred for this type of analysis in metal-rich giants, is not part of the Gaia-ESO spectra.

Second, there should likely be signs of magnetic activity in the Li-rich giants. Other candidates of Ap-type stars descendants were identified among giants and subgiants with magnetic activity (e.g., Aurière et al. 2014). However, fossil magnetic fields beneath the surface are hard to detect (Aurière et al. 2015; Stello et al. 2016). Nevertheless, the core-He burning star MG 340 is at one of the evolutionary phases where magnetic activity in giants is observed (Aurière et al. 2015). We checked the $\mathrm{H} \alpha$ line in our spectrum, but it shows no evident sign of activity. This is not inconsistent, as not all active giants display emission in $\mathrm{H} \alpha$ (see, e.g., Fekel \& Balachandran 1993). Emission should be clearer in the $\mathrm{Ca} \mathrm{H}$ and $\mathrm{K}$ lines or in the UV, which are not part of our spectra.

Finally, if such a scenario of extra-mixing inhibition is correct, it would likely apply to many, if not all, Li-rich giants with $A(\mathrm{Li}) \sim 1.50$ (or slightly higher) and masses between $\sim 1.5$ and 3.6 $M_{\odot}$. They would not have experienced fresh Li production, but would instead have preserved part of their original $\mathrm{Li}$ abundance.

Acknowledgements. We thank the anonymous referee for his/her suggestions and very fast report. R.S. acknowledges support by the National Science Center of Poland through grant 2012/07/B/ST9/04428. S.V. gratefully acknowledges the support provided by FONDECYT reg. n. 1130721. G.T. acknowledges support by the Research Council of Lithuania (MIP-082/2015). D.G. gratefully acknowledges support from the Chilean BASAL Centro de Excelencia en Astrofísica y Tecnologías Afines (CATA) grant PFB-06/2007. S.G.S. acknowledges the support from FCT through Investigador FCT contract of reference IF/00028/2014. E.D.M. acknowledges the support from FCT in the form of the grant SFRH/BPD/76606/2011. S.G.S. and E.D.M. also acknowledge the support from FCT through the project PTDC/FIS-AST/7073/2014. Based on data products from observations made with ESO Telescopes at the La Silla Paranal Observatory under program ID 188.B-3002. These data products have been processed by the Cambridge Astronomy Survey Unit (CASU) at the Institute of Astronomy, University of Cambridge, and by the FLAMES/UVES reduction team at INAF/Osservatorio Astrofisico di Arcetri. These data have been obtained from the Gaia-ESO Survey Data Archive, prepared and hosted by the Wide Field Astronomy Unit, Institute for Astronomy, University of Edinburgh, which is funded by the UK Science and Technology Facilities Council. This work was partly supported by the European Union FP7 program through ERC grant number 320360 and by the Leverhulme Trust through grant RPG-2012541. We acknowledge the support from INAF and Ministero dell' Istruzione, dell' Università' e della Ricerca (MIUR) in the form of the grant "Premiale VLT 2012" and "The Chemical and Dynamical Evolution of the Milky Way and Local Group Galaxies". The results presented here benefit from discussions held during 
the Gaia-ESO workshops and conferences supported by the ESF (European Science Foundation) through the GREAT Research Network Programme.

\section{References}

Adamów, M., Niedzielski, A., Villaver, E., Nowak, G., \& Wolszczan, A. 2012, ApJ, 754, L15

Adamów, M., Niedzielski, A., Villaver, E., Wolszczan, A., \& Nowak, G. 2014, A\&A, 569, A55

Alcalá, J. M., Biazzo, K., Covino, E., Frasca, A., \& Bedin, L. R. 2011, A\&A, 531, L12

Ashwell, J. F., Jeffries, R. D., Smalley, B., et al. 2005, MNRAS, 363, L81

Aurière, M., Lignières, F., Konstantinova-Antova, R., et al. 2014, in Putting A Stars into Context: Evolution, Environment, and Related Stars, eds G. Mathys, E. R. Griffin, O. Kochukhov, R. Monier, \& G. M. Wahlgren, 444

Aurière, M., Konstantinova-Antova, R., Charbonnel, C., et al. 2015, A\&A, 574, A90

Bharat Kumar, Y., Reddy, B. E., Muthumariappan, C., \& Zhao, G. 2015, A\&A, 577, A10

Böcek Topcu, G., Afşar, M., Schaeuble, M., \& Sneden, C. 2015, MNRAS, 446, 3562

Bressan, A., Marigo, P., Girardi, L., et al. 2012, MNRAS, 427, 127

Brown, J. A., Sneden, C., Lambert, D. L., \& Dutchover, Jr., E. 1989, ApJS, 71, 293

Cameron, A. G. W., \& Fowler, W. A. 1971, ApJ, 164, 111

Carlberg, J. K., Cunha, K., Smith, V. V., \& Majewski, S. R. 2012, ApJ, 757, 109

Carlberg, J. K., Smith, V. V., Cunha, K., \& Carpenter, K. G. 2016, ApJ, 818, 25

Carraro, G., Costa, E., \& Ahumada, J. A. 2010, AJ, 140, 954

Casey, A. R., Ruchti, G., Masseron, T., et al. 2016, MNRAS, submmitted [arXiv: 1603.03038]

Castilho, B. V., Spite, F., Barbuy, B., et al. 1999, A\&A, 345, 249

Charbonnel, C., \& Balachandran, S. C. 2000, A\&A, 359, 563

Charbonnel, C., \& Do Nascimento, Jr., J. D. 1998, A\&A, 336, 915

Charbonnel, C., \& Zahn, J.-P. 2007a, A\&A, 476, L29

Charbonnel, C., \& Zahn, J.-P. 2007b, A\&A, 467, L15

Christensen-Dalsgaard, J. 2015, MNRAS, 453, 666

de la Reza, R., Drake, N. A., \& da Silva, L. 1996, ApJ, 456, L115

de la Reza, R., Drake, N. A., Oliveira, I., \& Rengaswamy, S. 2015, ApJ, 806, 86

de Medeiros, J. R., Lebre, A., de Garcia Maia, M. R., \& Monier, R. 1997, A\&A, 321, L37

Dekker, H., D’Odorico, S., Kaufer, A., Delabre, B., \& Kotzlowski, H. 2000, in Optical and IR Telescope Instrumentation and Detectors, eds. M. Iye, \& A. F. Moorwood, SPIE Conf. Ser., 4008, 534

Delgado Mena, E., Tsantaki, M., Sousa, S. G., et al. 2016, A\&A, 587, A66

Denissenkov, P. A., \& Weiss, A. 2000, A\&A, 358, L49

Donati, P., Cantat Gaudin, T., Bragaglia, A., et al. 2014, A\&A, 561, A94

D’Orazi, V., Gratton, R. G., Angelou, G. C., et al. 2015, ApJ, 801, L32

Drake, N. A., de la Reza, R., da Silva, L., \& Lambert, D. L. 2002, AJ, 123, 2703

Fekel, F. C., \& Balachandran, S. 1993, ApJ, 403, 708

Garaud, P., \& Brummell, N. 2015, ApJ, 815, 42

Gilmore, G., Randich, S., Asplund, M., et al. 2012, The Messenger, 147, 25

Girardi, L. 1999, MNRAS, 308, 818

Girardi, L., Mermilliod, J.-C., \& Carraro, G. 2000, A\&A, 354, 892

Gonzalez, O. A., Zoccali, M., Monaco, L., et al. 2009, A\&A, 508, 289
Greenstein, J. L., \& Richardson, R. S. 1951, ApJ, 113, 536

Grevesse, N., Asplund, M., \& Sauval, A. J. 2007, Space Sci. Rev., 130, 105

Hill, V., \& Pasquini, L. 1999, A\&A, 348, L21

Jasniewicz, G., Parthasarathy, M., de Laverny, P., \& Thévenin, F. 1999, A\&A, 342,831

Kirby, E. N., Fu, X., Guhathakurta, P., \& Deng, L. 2012, ApJ, 752, L16

Kirby, E. N., Guhathakurta, P., Zhang, A. J., et al. 2016, ApJ, 819, 135

Kriskovics, L., Kővári, Z., Vida, K., Granzer, T., \& Oláh, K. 2014, A\&A, 571, A74

Kumar, Y. B., Reddy, B. E., \& Lambert, D. L. 2011, ApJ, 730, L12

Lagarde, N., Decressin, T., Charbonnel, C., et al. 2012, A\&A, 543, A108

Lattanzio, J. C., Siess, L., Church, R. P., et al. 2015, MNRAS, 446, 2673

Lèbre, A., de Laverny, P., de Medeiros, J. R., Charbonnel, C., \& da Silva, L. 1999, A\&A, 345, 936

Lèbre, A., de Laverny, P., Do Nascimento, Jr., J. D., \& de Medeiros, J. R. 2006, A\&A, 450, 1173

Lèbre, A., Palacios, A., Do Nascimento, Jr., J. D., et al. 2009, A\&A, 504, 1011

Lind, K., Asplund, M., \& Barklem, P. S. 2009, A\&A, 503, 541

Maeder, A., Meynet, G., Lagarde, N., \& Charbonnel, C. 2013, A\&A, 553, A1

Martell, S. L., \& Shetrone, M. D. 2013, MNRAS, 430, 611

McSwain, M. V., \& Gies, D. R. 2005, ApJS, 161, 118

Melo, C. H. F., de Laverny, P., Santos, N. C., et al. 2005, A\&A, 439, 227

Mocák, M., Meakin, C. A., Müller, E., \& Siess, L. 2011, ApJ, 743, 55

Monaco, L., Villanova, S., Moni Bidin, C., et al. 2011, A\&A, 529, A90

Monaco, L., Boffin, H. M. J., Bonifacio, P., et al. 2014, A\&A, 564, L6

North, P. 1993, in IAU Colloq. 138: Peculiar versus Normal Phenomena in Atype and Related Stars, eds. M. M. Dworetsky, F. Castelli, \& R. Faraggiana, ASP Conf. Ser., 44, 577

Palacios, A., Charbonnel, C., \& Forestini, M. 2001, A\&A, 375, L9

Palacios, A., Talon, S., Charbonnel, C., \& Forestini, M. 2003, A\&A, 399, 603

Pasquini, L., Randich, S., \& Pallavicini, R. 2001, A\&A, 374, 1017

Pasquini, L., Randich, S., Zoccali, M., et al. 2004, A\&A, 424, 951

Pasquini, L., Koch, A., Smiljanic, R., Bonifacio, P., \& Modigliani, A. 2014, A\&A, 563, A3

Pereyra, A., Castilho, B. V., \& Magalhães, A. M. 2006, A\&A, 449, 211

Pilachowski, C. A., Sneden, C., Kraft, R. P., Harmer, D., \& Willmarth, D. 2000, AJ, 119, 2895

Platais, I., Melo, C., Quinn, S. N., et al. 2012, ApJ, 751, L8

Power, J., Wade, G. A., Hanes, D. A., Aurier, M., \& Silvester, J. 2007, in Physics of Magnetic Stars, eds. I. I. Romanyuk, D. O. Kudryavtsev, O. M. Neizvestnaya, \& V. M. Shapoval, 89

Randich, S., \& Gilmore, G. 2013, The Messenger, 154, 47

Rebull, L. M., Carlberg, J. K., Gibbs, J. C., et al. 2015, AJ, 150, 123

Reddy, B. E., Lambert, D. L., Hrivnak, B. J., \& Bakker, E. J. 2002, AJ, 123, 1993

Ruchti, G. R., Fulbright, J. P., Wyse, R. F. G., et al. 2011, ApJ, 743, 107

Sacco, G. G., Morbidelli, L., Franciosini, E., et al. 2014, A\&A, 565, A113

Sackmann, I.-J., \& Boothroyd, A. I. 1999, ApJ, 510, 217

Siess, L., \& Livio, M. 1999, MNRAS, 308, 1133

Silva Aguirre, V., Ruchti, G. R., Hekker, S., et al. 2014, ApJ, 784, L16

Smiljanic, R., Korn, A. J., Bergemann, M., et al. 2014, A\&A, 570, A122

Stello, D., Cantiello, M., Fuller, J., et al. 2016, Nature, 529, 364

Strassmeier, K. G., Carroll, T. A., Weber, M., \& Granzer, T. 2015, A\&A, 574, A31

Tautvaišienè, G., Drazdauskas, A., Mikolaitis, ك̌., et al. 2015, A\&A, 573, A55

Wallerstein, G., \& Sneden, C. 1982, ApJ, 255, 577 


\section{Appendix A: Additional tables}

Table A.1. Observational data for the stars of Trumpler 20.

\begin{tabular}{|c|c|c|c|c|c|c|c|}
\hline Star ID & Gaia-ESO ID & $\begin{array}{c}\text { RA } \\
\operatorname{deg}(\mathrm{J} 2000)\end{array}$ & $\begin{array}{c}\text { Dec } \\
\operatorname{deg}(\mathrm{J} 2000)\end{array}$ & $\begin{array}{c}V \\
\text { mag }\end{array}$ & $\begin{array}{c}(B-V) \\
\text { mag }\end{array}$ & $\begin{array}{c}\mathrm{RV} \\
\mathrm{km} \mathrm{s}^{-1}\end{array}$ & $S / N$ \\
\hline 63 & $12385807-6030286$ & 189.7420 & -60.5079 & 13.60 & 1.59 & -40.81 & 109 \\
\hline 129 & $12400109-6031395$ & 190.0046 & -60.5276 & 14.72 & 1.42 & -40.04 & 58 \\
\hline 203 & $12393740-6032568$ & 189.9059 & -60.5491 & 14.89 & 1.35 & -40.18 & 43 \\
\hline 227 & $12394385-6033165$ & 189.9328 & -60.5546 & 14.61 & 1.34 & -40.50 & 50 \\
\hline 246 & $12394897-6033282$ & 189.9541 & -60.5578 & 14.55 & 1.34 & -39.07 & 44 \\
\hline 287 & $12394688-6033540$ & 189.9454 & -60.5650 & 14.80 & 1.35 & -40.48 & 53 \\
\hline 292 & $12390409-6034001$ & 189.7671 & -60.5667 & 13.41 & 1.55 & -40.08 & 98 \\
\hline 340 & $12391577-6034406$ & 189.8157 & -60.5779 & 14.74 & 1.35 & -40.21 & 49 \\
\hline 346 & $12394418-6034410$ & 189.9341 & -60.5781 & 14.71 & 1.37 & -40.50 & 50 \\
\hline 399 & $12395973-6035072$ & 189.9990 & -60.5853 & 14.55 & 1.40 & -41.74 & 78 \\
\hline 429 & $12400116-6035218$ & 190.0048 & -60.5894 & 14.54 & 1.37 & -39.27 & 59 \\
\hline 430 & $12395566-6035233$ & 189.9820 & -60.5898 & 15.25 & 1.00 & -41.79 & 35 \\
\hline 468 & $12400754-6035445$ & 190.0315 & -60.5957 & 13.47 & 1.61 & -39.86 & 83 \\
\hline 505 & $12392698-6036053$ & 189.8625 & -60.6015 & 14.59 & 1.42 & -40.31 & 66 \\
\hline 542 & $12391200-6036322$ & 189.8000 & -60.6089 & 14.69 & 1.33 & -40.58 & 60 \\
\hline 582 & $12391113-6036528$ & 189.7964 & -60.6146 & 14.92 & 1.36 & -40.76 & 49 \\
\hline 591 & $12400449-6036566$ & 190.0188 & -60.6157 & 13.62 & 1.64 & -40.73 & 88 \\
\hline 638 & $12395554-6037268$ & 189.9815 & -60.6241 & 14.64 & 1.39 & -40.07 & 83 \\
\hline 679 & $12402227-6037419$ & 190.0929 & -60.6283 & 14.74 & 1.42 & -41.13 & 47 \\
\hline 724 & $12390709-6038056$ & 189.7796 & -60.6349 & 14.92 & 1.31 & -38.38 & 46 \\
\hline 768 & $12394514-6038258$ & 189.9382 & -60.6405 & 14.80 & 1.38 & -40.68 & 56 \\
\hline 770 & $12392584-6038279$ & 189.8577 & -60.6411 & 14.93 & 1.34 & -42.97 & 36 \\
\hline 781 & $12394475-6038339$ & 189.9365 & -60.6427 & 14.62 & 1.40 & -37.89 & 52 \\
\hline 787 & $12395424-6038370$ & 189.9761 & -60.6436 & 14.61 & 1.39 & -42.48 & 65 \\
\hline 791 & $12394596-6038389$ & 189.9415 & -60.6441 & 14.54 & 1.38 & -38.96 & 65 \\
\hline 794 & $12391002-6038402$ & 189.7918 & -60.6445 & 13.57 & 1.57 & -40.60 & 87 \\
\hline 795 & $12394742-6038411$ & 189.9476 & -60.6447 & 14.72 & 1.36 & -39.30 & 46 \\
\hline 827 & $12395654-6039012$ & 189.9856 & -60.6503 & 14.78 & 1.38 & -38.23 & 57 \\
\hline 835 & $12393781-6039051$ & 189.9076 & -60.6514 & 14.49 & 1.35 & -39.71 & 45 \\
\hline 858 & $12394307-6039193$ & 189.9295 & -60.6554 & 14.63 & 1.42 & -40.82 & 54 \\
\hline 885 & $12395711-6039335$ & 189.9880 & -60.6593 & 14.64 & 1.37 & -41.01 & 62 \\
\hline 894 & $12393131-6039423$ & 189.8805 & -60.6617 & 14.77 & 1.34 & -35.29 & 56 \\
\hline 911 & $12400259-6039545$ & 190.0108 & -60.6651 & 13.74 & 1.61 & -40.41 & 106 \\
\hline 923 & $12394121-6040040$ & 189.9217 & -60.6678 & 14.84 & 1.37 & -41.01 & 56 \\
\hline 950 & $12392636-6040217$ & 189.8599 & -60.6727 & 14.74 & 1.35 & -40.67 & 42 \\
\hline 1008 & $12394715-6040584$ & 189.9465 & -60.6829 & 13.85 & 1.57 & -39.41 & 78 \\
\hline 1010 & $12394049-6041006$ & 189.9188 & -60.6835 & 14.56 & 1.37 & -41.99 & 48 \\
\hline 1044 & $12400278-6041192$ & 190.0116 & -60.6887 & 14.95 & 1.38 & -38.75 & 40 \\
\hline 1082 & $12390478-6041475$ & 189.7699 & -60.6965 & 14.59 & 1.32 & -40.15 & 52 \\
\hline 2690 & $12383657-6045300$ & 189.6525 & -60.7583 & 14.57 & 1.44 & -41.62 & 27 \\
\hline 2730 & $12383597-6045242$ & 189.6498 & -60.7568 & 14.94 & 1.38 & -40.08 & 53 \\
\hline 3470 & $12402478-6043103$ & 190.1033 & -60.7195 & 13.92 & 1.60 & -39.84 & 57 \\
\hline
\end{tabular}

Notes. The star ID is taken from McSwain \& Gies (2005). The $V$ magnitude and $(B-V)$ color have been corrected from differential reddening by Donati et al. (2014). The radial velocities and signal-to-noise values were determined from the bluer part of the UVES spectrum as described in Sacco et al. (2014). 
A\&A 591, A62 (2016)

Table A.2. Atmospheric parameters and lithium abundances for the stars of Trumpler 20.

\begin{tabular}{|c|c|c|c|c|c|c|c|c|c|c|c|c|}
\hline Star ID & $\begin{array}{l}T_{\text {eff }} \\
(\mathrm{K})\end{array}$ & $\begin{array}{c}\sigma \\
(\mathrm{K})\end{array}$ & $\begin{array}{l}\log g \\
(\operatorname{dex})\end{array}$ & $\begin{array}{c}\sigma \\
(\operatorname{dex})\end{array}$ & $\begin{array}{c}{[\mathrm{Fe} / \mathrm{H}]} \\
(\mathrm{dex})\end{array}$ & $\begin{array}{c}\sigma \\
(\mathrm{dex})\end{array}$ & $\begin{array}{c}\xi \\
\mathrm{km} \mathrm{s}^{-1}\end{array}$ & $\begin{array}{c}\sigma \\
\mathrm{km} \mathrm{s}^{-1}\end{array}$ & $\begin{array}{l}A(\mathrm{Li})_{\mathrm{LTE}} \\
(\mathrm{dex})\end{array}$ & $\begin{array}{c}\sigma \\
(\operatorname{dex})\end{array}$ & Flag & $A(\mathrm{Li})_{\text {non-LTE }}$ \\
\hline 63 & 4551 & 133 & 2.18 & 0.29 & 0.09 & 0.11 & 1.45 & 0.06 & 0.50 & 0.38 & $\lim$. & 0.75 \\
\hline 129 & 4888 & 116 & 2.85 & 0.23 & 0.13 & 0.10 & 1.39 & 0.04 & 0.85 & 0.05 & lim. & 1.02 \\
\hline 203 & 5031 & 120 & 3.00 & 0.22 & 0.14 & 0.10 & 1.40 & 0.10 & 1.07 & 0.14 & lim. & 1.22 \\
\hline 227 & 5004 & 113 & 2.87 & 0.23 & 0.09 & 0.10 & 1.59 & 0.06 & 1.04 & 0.18 & lim. & 1.20 \\
\hline 246 & 4947 & 114 & 2.81 & 0.22 & 0.10 & 0.09 & 1.50 & 0.09 & 1.03 & 0.18 & lim. & 1.21 \\
\hline 287 & 4961 & 116 & 2.90 & 0.23 & 0.14 & 0.09 & 1.38 & 0.06 & 0.87 & 0.12 & lim. & 1.03 \\
\hline 292 & 4406 & 114 & 2.05 & 0.24 & 0.00 & 0.10 & 1.55 & 0.09 & 0.28 & 0.34 & $\lim$. & 0.60 \\
\hline 340 & 4851 & 118 & 2.88 & 0.23 & 0.02 & 0.10 & 1.36 & 0.06 & 1.37 & 0.21 & det. & 1.54 \\
\hline 346 & 4963 & 118 & 2.81 & 0.23 & 0.15 & 0.10 & 1.47 & 0.13 & 0.92 & 0.14 & lim. & 1.08 \\
\hline 399 & 4876 & 113 & 2.76 & 0.22 & 0.10 & 0.10 & 1.41 & 0.06 & 0.77 & 0.22 & lim. & 0.95 \\
\hline 429 & 4887 & 122 & 2.77 & 0.22 & 0.10 & 0.10 & 1.38 & 0.05 & 0.70 & 0.10 & lim. & 0.87 \\
\hline 430 & 5992 & 125 & 3.79 & 0.25 & 0.20 & 0.10 & 1.64 & 0.21 & 2.83 & 0.21 & det. & 2.81 \\
\hline 468 & 4435 & 115 & 2.10 & 0.23 & 0.06 & 0.10 & 1.52 & 0.05 & 0.34 & 0.31 & lim. & 0.65 \\
\hline 505 & 4834 & 120 & 2.76 & 0.25 & 0.11 & 0.10 & 1.39 & 0.10 & 1.07 & 0.21 & det. & 1.25 \\
\hline 542 & 4939 & 112 & 2.83 & 0.23 & 0.15 & 0.10 & 1.33 & 0.05 & 0.93 & 0.20 & lim. & 1.09 \\
\hline 582 & 4967 & 115 & 2.93 & 0.23 & 0.18 & 0.10 & 1.39 & 0.11 & 0.97 & 0.15 & lim. & 1.13 \\
\hline 591 & 4412 & 132 & 2.14 & 0.25 & 0.00 & 0.10 & 1.59 & 0.23 & 1.32 & 0.21 & det. & 1.60 \\
\hline 638 & 4900 & 112 & 2.79 & 0.22 & 0.13 & 0.10 & 1.38 & 0.05 & 0.74 & 0.20 & lim. & 0.91 \\
\hline 679 & 4936 & 121 & 2.77 & 0.23 & 0.12 & 0.10 & 1.50 & 0.10 & 0.92 & 0.15 & lim. & 1.08 \\
\hline 724 & 5026 & 120 & 3.14 & 0.23 & 0.10 & 0.10 & 1.27 & 0.04 & 1.01 & 0.13 & lim. & 1.15 \\
\hline 768 & 4928 & 119 & 2.85 & 0.23 & 0.12 & 0.10 & 1.36 & 0.05 & 0.85 & 0.15 & lim. & 1.01 \\
\hline 770 & 5035 & 119 & 3.14 & 0.25 & 0.12 & 0.10 & 1.28 & 0.07 & 1.15 & 0.14 & lim. & 1.29 \\
\hline 781 & 4882 & 118 & 2.77 & 0.23 & 0.12 & 0.10 & 1.48 & 0.09 & 0.88 & 0.18 & lim. & 1.05 \\
\hline 787 & 4913 & 109 & 2.80 & 0.23 & 0.14 & 0.10 & 1.42 & 0.04 & 0.90 & 0.22 & lim. & 1.07 \\
\hline 791 & 4889 & 122 & 2.75 & 0.24 & 0.10 & 0.11 & 1.42 & 0.06 & 0.70 & 0.13 & lim. & 0.87 \\
\hline 794 & 4471 & 118 & 2.14 & 0.23 & 0.01 & 0.10 & 1.56 & 0.17 & 0.43 & 0.05 & lim. & 0.74 \\
\hline 795 & 4924 & 117 & 2.77 & 0.23 & 0.08 & 0.10 & 1.50 & 0.07 & 0.96 & 0.17 & lim. & 1.13 \\
\hline 827 & 4932 & 122 & 2.83 & 0.25 & 0.16 & 0.10 & 1.39 & 0.05 & 0.89 & 0.17 & lim. & 1.05 \\
\hline 835 & 4935 & 128 & 2.79 & 0.22 & 0.12 & 0.11 & 1.44 & 0.14 & 0.87 & 0.11 & lim. & 1.03 \\
\hline 858 & 4880 & 117 & 2.76 & 0.23 & 0.12 & 0.09 & 1.34 & 0.05 & 0.69 & 0.11 & lim. & 0.87 \\
\hline 885 & 4964 & 116 & 2.83 & 0.23 & 0.14 & 0.09 & 1.44 & 0.06 & 0.93 & 0.05 & lim. & 1.09 \\
\hline 894 & 4988 & 114 & 3.04 & 0.22 & 0.13 & 0.10 & 1.37 & 0.03 & 0.95 & 0.16 & lim. & 1.10 \\
\hline 911 & 4521 & 115 & 2.20 & 0.22 & 0.03 & 0.09 & 1.47 & 0.15 & 0.50 & 0.39 & lim. & 0.76 \\
\hline 923 & 4989 & 117 & 2.94 & 0.25 & 0.13 & 0.09 & 1.41 & 0.03 & 0.96 & 0.17 & lim. & 1.11 \\
\hline 950 & 4953 & 121 & 2.87 & 0.23 & 0.08 & 0.10 & 1.47 & 0.23 & 1.06 & 0.22 & det. & 1.22 \\
\hline 1008 & 4586 & 123 & 2.29 & 0.24 & 0.05 & 0.10 & 1.49 & 0.08 & 0.43 & 0.05 & lim. & 0.67 \\
\hline 1010 & 4926 & 120 & 2.82 & 0.24 & 0.10 & 0.11 & 1.40 & 0.10 & 0.94 & 0.16 & lim. & 1.11 \\
\hline 1044 & 4951 & 117 & 2.94 & 0.22 & 0.12 & 0.09 & 1.34 & 0.10 & 0.97 & 0.05 & lim. & 1.13 \\
\hline 1082 & 5003 & 116 & 2.95 & 0.22 & 0.18 & 0.09 & 1.47 & 0.07 & 1.00 & 0.16 & lim. & 1.15 \\
\hline 2690 & 4689 & 128 & 2.52 & 0.25 & -0.01 & 0.12 & 1.37 & 0.06 & 0.68 & 0.05 & lim. & 0.90 \\
\hline 2730 & 5003 & 117 & 2.97 & 0.24 & 0.14 & 0.09 & 1.51 & 0.08 & 0.99 & 0.17 & lim. & 1.15 \\
\hline 3470 & 4590 & 123 & 2.28 & 0.22 & 0.03 & 0.11 & 1.48 & 0.10 & 0.51 & 0.18 & lim. & 0.75 \\
\hline
\end{tabular}

Notes. The star ID is taken from McSwain \& Gies (2005). For the metallicity, we adopt $A(\mathrm{Fe})_{\odot}=7.45$ as solar reference (Grevesse et al. 2007). The flag indicates if the $\mathrm{Li}$ abundance is a detection or an upper limit. 